\title{
PROFESORES, REFORMAS Y PARTICIPACIÓN
}

\author{
Luis Reyes Ochoa \\ Celia Fernandes Nunes ${ }^{* * 4}$ \\ Rodrigo Fuentealba Jara ${ }^{\text {t*at }}$
}

\begin{abstract}
RESUMEN
Los constantes cambios acaecidos en los últimos decenios en las sociedades latinoamericanas y en el mundo, han producido profundas transformaciones que han tenido como efecto una reorganización económica, en el marco de la globalización y la instalación de la Sociedad del Conocimiento. Los procesos anteriores han generado reformas educativas, que han afectado directamente el desempeño profesional del profesor en su contexto laboral. Observando con atención las propuestas de reformas educacionales en los distintos países, se ha percibido que los profesores han sido los grandes ausentes. En este artículo, se procura analizar brevemente las características de las propuestas educacionales implementadas en Brasil y Chile, a partir de lo cual se analiza cuál ha sido la participación de los profesores y qué tipo de contribuciones ellos han podido hacer en la elaboración e implementación de tales propuestas educativas.
\end{abstract}

Palabras Clave: Profesores y Reformas Educativas

\section{TEACHERS, REFORM AND PARTICIPATION}

ABSTRACT

The economic reorganization in the context of globalization and the installation of the knowledge society have created educational reforms which have directly affected the professional performance of teachers in their work context. By carefully observing the proposals for educational reforms in different countries, it has been apparent that teachers have been notably absent in developing these proposal. In this article, the authors seek to briefly analyze the characteristics of educational proposals implemented in Brazil and Chile, from which, they examine what has been the involvement of teachers and what kind of contributions they have done in the development and implementation of such educational proposals.

Keywords: Teachers and educational reform.

* Profesor y Magister en Educación. Académico de Departamento de Educación, Universidad Católica Silva Henríquez. Santiago de Chile. 1reyeso@ucsh.cl

* Doutora en Educação. Professora adjunta da Universidade Federal de Ouro Preto, Minas Gerais, Brasil.celia@ichs.ufop.br

Doctor en Ciencias de la Educación. Académico de Departamento de Educación, Universidad Católica Silva Henríquez. Santiago de Chile.rfuentea@ucsh 


\section{Reformas Educativas en América Latina}

Los últimos años se han caracterizado por la implementación del proyecto neoliberal en varios países, incluidos los de América Latina, como modelo hegemónico de una ideología mundial. Según algunos autores que investigan esta temática, este proyecto fue consolidado en los países capitalistas desarrollados en la década de los '70, basado en la implementación de propuestas de economía clásica como camino para la superación de la crisis de esas sociedades. Es característica del neoliberalismo, que el Estado tenga una mínima intervención en la economía y no controle las actividades económicas privadas, posibilitando así la libre imposición de los mecanismos del mercado internacional. En este sentido, para comprender cómo este proyecto liberal ha percibido la cuestión educacional, es importante, en época de globalización, entenderlo como un proceso inserto en un contexto dinámico mayor (Bianchetti, 1997).

Este proyecto posee como característica la creación de categorías propias de percepción de la sociedad y del mundo, y prioriza una doble visión. Por un lado, implementando estrategias políticas, económicas y jurídicas para enfrentar la crisis capitalista; y por otro vehiculando una ideología donde sólo existe una determinada realidad divulgada en un nuevo sentido común, siendo imposible la elaboración de otra cuando se intenta promover el desarrollo de una economía equilibrada. De esa forma todos deben adaptarse a sus reglas. Anderson hace un análisis de este fenómeno hegemónico y concluye, que:

Económicamente, el neoliberalismo fracasó, no consiguiendo ninguna revitalización básica del capitalismo avanzado. Socialmente, al contrario, el neoliberalismo consiguió muchos de sus objetivos, creando sociedades marcadamente más desiguales, aunque no tan desestatizadas como quería. Política e ideológicamente, sin embargo, el neoliberalismo alcanzó éxito en un grado con el cual sus fundadores jamás soñaron, diseminando la simple idea de que no hay alternativas para sus 
principios, que todos, sea confesando o negando, tienen que adaptarse a sus normas. (Anderson, 1995: p. 23).

Aunque el referido autor destaque el fracaso económico de las políticas neoliberales, se percibe, en la práctica, una sobrevaloración de esta área sobre las otras esferas de la vida humana, siendo este aspecto esencial en el análisis de las relaciones sociales, políticas, educativas entre otras, que de una forma o de otra, colaboran en la producción de una sociedad más desintegrada y desigual. Lo anterior ha contribuído a una desestructuración de la representación colectiva de los intereses populares y a un desestímulo a los movimientos sociales organizados. Estos hechos son recurrentes en la inculcación de los elementos centrales de este proyecto global que se ha caracterizado a través de la construcción de la política como manipulación del afecto y del sentimiento; la transformación del espacio de discusión política en estrategias de convencimiento publicitario; la celebración de la supuesta eficiencia y productividad de la iniciativa privada en oposición a la ineficacia y al desperdicio de los servicios públicos; la redefinición de la ciudadanía cual agente político se transforma en agente económico y el ciudadano en consumidor.

En el contexto del modelo neoliberal, se ha evidenciado que los problemas del sistema educativo son producto de una gestión deficitaria que no garantiza la participación en una competitividad globalizada. La gestión, entonces, es vista como el principal aspecto a ser considerado en la reforma administrativa del sistema escolar, como forma de promover estrategias de control que posibiliten la calidad de la enseñanza. La principal discusión en torno a la educación es de que ésta solamente será de calidad a partir del control y de la evaluación de los servicios educativos y de la articulación de la formación escolar con los objetivos del mercado de trabajo. De tal manera que queda planteado un desafío para la educación que deberá además de instrumentalizar al individuo para la productividad, desarrollar una formación política para el alcance de la ciudadanía. En este sentido, ahora la meta es que, a través de la educación, pueda prepararse mano de obra calificada, no tan especializada como anteriormente, 
pero con una formación general y flexible de calidad para atender la competitividad del mercado. A partir de esos cambios, es de esperar que ocurra también una modificación en el sistema escolar, de forma de atender a esa nueva demanda de formación de mano de obra para el mercado.

Además de lo anterior, uno de los cambios significativos de esta época, es la conciencia de que estos países se están convirtiendo, gradualmente, en una sociedad multiétnica y pluricultural. Esta realidad, unida a los procesos migratorios, desafía a cada ciudadano a aprender y practicar los valores del respeto mutuo, del diálogo sincero y tolerante. Ya que la sociedad globalizada en la que se está inmerso actualmente, lleva a establecer relaciones distintas en lo político, en lo económico, en lo social, en lo religioso, con nuevas oportunidades de comunión y mutuo conocimiento, pero paradójicamente, con profundas soledades, como consecuencia de actitudes individualistas que nacen del egoísmo ${ }^{\mathrm{I}}$.

En este contexto de mutación cultural, los desafíos que se comportan a la educación son cada vez más complejos. Y es por eso que la educación formal e institucionalizada tiene un rol clave que desarrollar y cumplir específicamente en lo relacionado a promover un compromiso social y cívico por parte de los jóvenes.

Los requerimientos que la sociedad moderna hace a los sistemas educativos han sido largamente estudiados, investigados y analizados por organismos en el ámbito nacional, regional y mundial. Todos los diagnósticos son coincidentes al determinar que, junto con mejorar la calidad de las prácticas pedagógicas de los profesores en las salas de clases, se impone realizar una revisión, actualización y renovación de la gestión de las unidades educativas.

Con relación a la calidad de educación ha surgido la necesidad de implementar, paulatinamente desde el año 1990, procesos de intervención con el objetivo de elevar los resultados en los aprendizajes

I Conferencia Episcopal de Chile: Orientaciones Pastorales 200I-2005. 
de los alumnos, expresados en las mediciones estandarizadas. Sin lugar a dudas, los cambios educativos necesitan una serie de innovaciones en los más diversos niveles, tales como la modificación de las normas e instrumentos técnicos, la flexibilidad curricular, el perfeccionamiento de los docentes en ejercicio, entre otros. Pero dichos cambios pasan fundamentalmente por las prácticas de enseñanza-aprendizaje que los profesores llevan a cabo con sus estudiantes. Es en este proceso y en las interacciones formativas situadas en la sala de clase y en las escuelas, donde podría situarse el cambio educacional.

La propuesta educativa en curso enfatiza no el rol del profesor o del proceso de enseñanza, sino el rol del estudiante y su aprendizaje, pero sin descuidar el polo antes enunciado, sino más bien se enfatiza el proceso de enseñanza y aprendizaje en cuanto relación didáctica ${ }^{2}$. Lo que se releva entonces es que los distintos estamentos relacionados con la acción educativa, deben asumir el reto de dar respuesta a las necesidades del desarrollo de los estudiantes, propiciando espacios válidos para el aprendizaje. La descentralización curricular permite una diversidad de respuestas curriculares. Por lo tanto, la diversidad no puede ofrecerse desde un programa cerrado, sino desde una propuesta flexible que posibilite la inclusión de todas las peculiaridades.

Este nuevo escenario reclama un cambio profundo en los procesos evaluativos y en la búsqueda de estrategias didácticas que faciliten la atención a los diversos ritmos y estilos de aprendizajes, de tal forma que posibilite un aprendizaje más significativo, que efectivamente centre la mirada en el desarrollo de las capacidades superiores de los alumnos y los haga protagonistas de su propio aprendizaje. Ante estas necesidades es relevante destacar que, dentro de los muchos factores que intervienen en el proceso de enseñanza aprendizaje, la motivación es uno de los más importantes, puesto que afecta el aprendizaje, porque es un desencadenante de conductas que estimulan al estudiante tanto fisiológica como psicológicamente, además de proveer de objetivos hacia los cuales se dirigen las acciones.

2 Ministerio de Educación (2004): Marco para la buena enseñanza. 


\section{La participación de los profesores en las Reformas Educativas}

Las nuevas demandas en el campo social y educacional, configuran un nuevo escenario en el cual se obliga a los profesores y profesoras a trabajar en cierto sentido de manera inédita con respecto a su experiencia docente anterior. La realidad de los alumnos se complejiza tanto en los países desarrollados como en los países en desarrollo, aunque los problemas no sean del todo comparables. Se emiten mensajes que apuntan a la responsabilidad del profesor o profesora por el aprendizaje de sus alumnos y se busca medir su éxito según resultados. Paralelamente a esta creciente tendencia, aparece una construcción teórica con relación a la profesión docente, sobre la base de estudios cualitativos que muestran la importancia de los procesos mentales o pensamiento del profesor, según los cuales los profesores procesan la ejecución de su docencia o, acceden o rechazan las reformas educacionales propuestas. Los estudios muestran, también, la ineficacia de muchos de los sistemas tradicionales de perfeccionamiento y revelan la actitud de cuestionamiento de profesores y profesoras respecto a los "expertos" que las conducen.

Aun sin haberse consolidado suficientemente una cultura de aprendizaje colegiado entre los profesores y profesoras, que hubiera permitido una transición desde una enseñanza formalista a una etapa de mayor innovación, presenciamos el surgimiento de otras concepciones relacionadas con las nuevas demandas de la globalización de los procesos culturales y económicos y la sociedad de la información. Las demandas que surgen en este contexto, ya no son solamente lograr los aprendizajes tradicionales de la escuela, sino manejar e interpretar contenidos y estrategias didácticas de acuerdo a los contextos de desempeño, propuestas curriculares genéricas y hasta cierto punto ambiguas y estimular el desarrollo de habilidades cognitivas de muy distinto orden a lo que ya estaban acostumbrados. Al respecto, no hay muchos modelos, lo que sí está claro es que no basta la ayuda del colega para traducir y rediseñar las tareas de aprendizaje. 
Aunque las políticas educativas reconozcan el papel fundamental que el profesor tiene en el proceso educativo, no consideran adecuadamente la importancia de lidiar con la cuestión docente de un modo más amplio, priorizando sólo la inversión en la formación, dejando de lado las condiciones de vida, de trabajo y de la historia de la profesión.

Así, al relacionar el problema de la calidad de la enseñanza al profesor, las propuestas educativas han optado por preocuparse exclusivamente de la formación de éste. En su propuesta, reconoce que sin el profesor no es posible hablar de calidad, y como estrategia de inversión buscar entrenar al profesor y controlar su desempeño a través de evaluación. Así, para atender la cuestión de la formación docente se ha buscado invertir en la capacitación en servicio, a través del ofrecimiento de perfeccionamientos en diferentes modalidades (presencial y o/a la distancia) con el objetivo de repasar contenidos que mejoren la práctica pedagógica. Sin embargo, en el análisis de tales cursos han sido caracterizados como excesivamente centralizados tanto en su planificación como en su aplicación; así, van configurándose como paquetes cerrados de perfeccionamiento (definidos siempre por equipos de técnicos, expertos y hasta consultores de empresas) planificados de forma centralizada, sin participación de los grupos de profesores involucrados en el proceso de formación, y presentando una alta transferibilidad (o sea, con gran potencial para que sean aplicados en diferentes contextos geográficos y con diferentes poblaciones).” (Gentili, 1996).

La literatura en el área, ya viene mostrándonos la ineficacia de esos cursos de perfeccionamiento, que no se traducen en recursos de cambio efectivo en la práctica pedagógica. Esto, porque ellos, generalmente son impuestos por agentes que están fuera de la escuela, no considerando los anhelos e intereses de capacitación solicitados por los profesores. Aun así, aunque las políticas educativas reconocen la importancia del profesor en relación a la calidad y para esto hagan inversión en su formación, los profesores aún no han tenido su papel debidamente reconocido. 
Esa no consideración del papel del profesor se ha manifestado bajo dos formas; en primer lugar, se percibe una ausencia de participación docente en las discusiones y decisiones de políticas educativas y en un segundo momento, la elaboración de esas propuestas queda bajo la responsabilidad de economistas, quedándose la ejecución de las mismas por cuenta de los profesores, relegando a estos últimos a un papel secundario, ejecutor y pasivo.

En este sentido, en un análisis de las medidas implementadas en el área educativa por el Banco Mundial (Torres, 1996), se concluye que éstas a pesar de recientes, están de cierta forma reproduciendo un modelo educativo convencional. Y destaca que, con relación al profesor, esas políticas muestran una gran incomprensión y negligencia al lidiar con la cuestión docente, con las condiciones de vida y de trabajo de los docentes, su perfil profesional y su formación continua, desconociendo el hecho de que los profesores son actores esenciales en la calidad y en el cambio en educación. Se desplaza al docente a un papel secundario y pasivo, no considerando la participación y la consulta del magisterio como condición fundamental en el diseño, ejecución y eficacia de las políticas y en particular de la reforma educativa; se evidencia una visión sesgada y de corto plazo de la formación docente, y concibe la capacitación como un problema de adecuación de los docentes a fines y objetivos pre establecidos; privilegia en el docente su conocimiento y su actualización en contenidos, diminuyendo la importancia de su formación pedagógica (Torres, 1996).

Otro autor, también identifica este problema de la ausencia de participación de profesores en las decisiones políticas, lo que tal vez posibilitaría un análisis crítico de la naturaleza y del proceso de las reformas educativas (Giroux, 1997). Alerta que tales cambios educativos acaban siendo percibidos por el profesor, como una amenaza recurrente de la falta de confianza en la capacidad de ellos mismos. El autor resalta que:

...muchas de las recomendaciones que surgieron en el actual debate, ignoran el papel que los profesores desempeñan en la 
preparación de aprendices para que sean ciudadanos activos y críticos, o entonces sugieren reformas que ignoran la inteligencia, juicio y experiencia que los profesores podrían ofrecer en tal debate. Cuando los profesores de hecho entran en el debate es para que sean objetos de reformas educativas que los reducen al estatus de técnicos de alto nivel, cumpliendo dictámenes y objetivos decididos por especialistas un tanto alejados de la realidad cotidiana de la vida del aula (Giroux, 1997: p. 157).

En un estudio que va más allá que simplemente responsabilizar al profesor por la falta de calidad en la escuela (Esteve, I995), se analiza cómo queda la situación de éstos delante de los problemas del cotidiano escolar resultantes de los cambios implementados. El autor resalta que mediante los problemas que van apareciendo en la escuela, el profesor intenta encontrar soluciones con el fin de resolverlos. En este sentido, Esteve dice que el problema reside en que, independientemente de quién provocó el cambio, son los profesores los que dan la cara. Son ellos, por lo tanto, quienes tienen que encontrar una salida airosa, aunque no sean los responsables. Las reacciones ante esta situación son muy variadas; pero en cualquier caso, la palabra malestar podría resumir los sentimientos de este grupo de profesores ante una serie de circunstancias imprevistas que los obligan a hacer un papel ridículo, ya que ellos enfrentan circunstancias de cambio que los obligan a hacer apenas su trabajo, teniendo que soportar la crítica generalizada que, sin analizar esas circunstancias, los considera como responsables inmediatos por los fracasos del sistema de enseñanza (Esteve, 1995).

En este sentido, es importante también considerar las soluciones que son encontradas por los profesores ante las situaciones a las cuales se enfrentan, al contrario de solamente responsabilizarlo por los problemas educativos. El reconocimiento de esta cuestión, posibilitaría un volver a pensar el papel de los profesores y la proposición de alternativas a fin de atender los cambios exigidos en el contexto educativo actual. 


\section{Profesores y Reforma Educativa en Brasil}

Al observar la realidad del país más grande de América del Sur, Brasil, se evidencia que ha existido una gran inversión pública en el área educativa a través del aumento de la oferta de escuelas y vacantes en las escuelas públicas, acompañadas de varias reformas educativas en todos los niveles. No obstante, lo anterior se ha analizado por los profesionales de la educación como insuficiente, porque en el tiempo no se ha presentando una mejora significativa sobre la educación o incluso un progreso en el trabajo del maestro.

Las transformaciones educacionales implementadas han buscado, sobre la justificación de una ineficacia de la escuela pública, llevar a cabo las orientaciones en dos sentidos: por una parte, a través de la descentralización de aspectos administrativo-financieros que buscan la autonomía escolar; y, por otra, en la gestión en los aspectos del cotidiano escolar como la definiciones curriculares de indicadores; los programas de la evaluación; la oferta de programas de formación educativa, los programas de libros de texto, entre otros. Es evidente que las reformas en la educación brasileña en los últimos años presentaron las características de regulación y control, causando un impacto en el trabajo del maestro.

En este contexto, el papel del maestro va siendo redefinido en la perspectiva de la reestructuración del trabajo educativo. El maestro, debido a las funciones variadas que la escuela pública asume, tiene que abordar otras dimensiones de su formación. En el caso de Brasil, como en algunos países de América Latina, comienza una inversión en los programas de formación continua de los maestros que se consideran como un aspecto crucial de las reformas educativas. También se nota una intensificación de proyectos dirigidos a la formación de los maestros que ellos necesitan para estar calificados para ayudar en las nuevas demandas profesionales y las demandas de la escuela. Se señala que los profesores, son muy buscados por los programas gubernamentales como los agentes centrales del cambio en los momentos de reforma educativa, siendo considerados 
los principales responsables por la acción de los estudiantes, de la escuela y del sistema. Antes de ese escenario, a los maestros por mucho tiempo los reprimieron, haciéndoles a ellos tomar para sí mismos la responsabilidad por el éxito o fracaso de los programas (Oliveira, 2003).

En una investigación realizada acerca de la escuela pública, cuando se analizaron las dificultades que las mismas pueden reconocer, están los cambios constantes que se proponen por los órganos superiores y ese excesivo propósito de influenciar en el trabajo del maestro, como señala un director, se plantea que cuando ellos cambian a los gobiernos, ellos cambian las concepciones, de tal manera que el profesor tiene que innovar (Nunes, 2004).

Todavía, según un director, la forma de administración y comunicación usada por los órganos superiores, es considerada como una dificultad que se enfrenta en el cotidiano de la gestión educativa, por consiguiente la mayoría de las veces las ordenanzas, leyes y las reglas terminan llegando a la escuela a través del periódico, sin cualquier advertencia administrativa anterior.

Nosotros los directores nos informamos por el periódico... cuando llega a nosotros la información... la cosa ya pasó, ya aconteció, y nosotros tenemos que estar corriendo detrás de... Yo pienso que cuando ellas fueron publicadas en el periódico, inmediatamente el director tenía que estar informado... para eso es el empleado de él. Entonces lo que yo pienso es que es una dificultad. Porque la ley cada uno la entiende de una manera... dictó una ley que habla sobre el premio de las vacaciones ahora. Yo fui detrás de la inspectora a hablar con ella. Ella dijo: yo no hablaré con usted, porque la ley cada uno la entiende de una manera. Y nosotros no tenemos ningún estudio. Entonces yo tengo que pasar alguna información para mi maestro, porque él tuvo noticias de la boca de otro. Y yo tampoco puedo pasar nada... Yo encuentro muy difícil... Y parece que el director, él no sabe nada... (Nunes, 2004: p. 65). 
La queja constante es, que los cambios presentados por los órganos superiores del sistema educativo de una cierta manera, en la mayoría de los casos acaban tomando por sorpresa a los maestros, porque ellos no son considerados en las discusiones previas y los cambios no vienen estructurados por los profesores para que los puedan entender. Esto es así, el director normalmente hace, elabora los proyectos, determina las leyes y los instrumentos escolares.

Investigaciones desarrolladas con directores y sub-directores del sector público revelaron que situaciones como éstas, han estado generando en estos profesionales una cierta impotencia antes de los cambios (Oliveira, 2002). Muchas veces ellos no consiguen responder a las demandas de los plazos determinados por los órganos centrales, provocando y sintiéndose disconformes, provocando en los directores que se sientan amenazados e inhibidos para tomar decisiones, ya sea por las limitaciones de la infraestructura o por el miedo de que ellos sean interpretados como líderes autoritarios.

Entre esos cambios constantes, el director resalta, por ejemplo, la implantación del régimen de evaluación continua a través del sistema de ciclos en la enseñanza fundamental. Esto puede ser considerado como una actitud drástica impuesta por las reformas educativas, porque generó problemas como: la resistencia entre los profesionales de la educación, cambios radicales entre la enseñanza y el aprendizaje y la evaluación sin cambiar las bases eficaces para eso (como capacitarse, condiciones materiales de la escuela, inversión en infraestructura, entre otros); los problemas referidos a la disciplina que acarrea una impotencia en la práctica del maestro (Oliveira, 1999).

Esa falta de adhesión de los profesionales de la educación en la implantación del proyecto de ciclos, puede ser considerado como una de las justificaciones por el poco éxito que tuvo este proyecto. Para otro autor, estos profesionales, principalmente los maestros, ellos son los elementos centrales para el éxito de proyectos y los programas educativos, porque ellos son los que de hecho los ponen en la práctica. Se requiere la participación amplia de los profesionales en la formulación y en la adaptación de propuestas de ciclos, así como 
en el proceso de evaluación permanente de la propuesta (Mainardes, 200I).

Dentro de las acciones de las reformas educacionales e implementación de sistemas de evaluación en todos los niveles, generó un gran impacto en la escuela y en el trabajo del maestro. Esas acciones para hacer una contribución al logro de un diagnóstico del sistema educativo, fueron elaboradas muchas veces sin considerar los aspectos pertinentes al proceso de la enseñanza-aprendizaje (social y cultural, entre otros).

Analizando esos programas especiales implantados por la política educativa, se enfatiza que esos cambios en las formas de evaluación requieren del maestro, además de un dominio mayor de conocimiento, un cambio de conducta (Oliveira 2000). Ello todavía aumenta otros factores que influencian esa realidad, como la necesidad de más tiempo para la preparación de las clases y el número de estudiantes por grupo, así como las condiciones estructurales de las escuelas y los contratos de los trabajos de los maestros.

Considerando lo antes expuesto, analizamos que muchos de los fracasos de las reformas educativas nacen de que son planificadas sin considerar las concepciones básicas de las escuelas y de los actores que viven la práctica escolar.

Lo que se percibe es que, ante las reformas que ellos impusieron, las escuelas reaccionan diferentemente, algunas rechazándolas, intentando acomodarse o adaptarlas a las necesidades reales, o, todavía, asimilándolas con la substitución y renovación de sus concepciones y valores (Teixeira, 1999).

Se puede afirmar que es un proceso que desnaturaliza esas cosas inevitables de las reformas educativas, debiéndose buscar un proceso democrático de reformas y de políticas educacionales que valoren la participación educativa en los aspectos políticos, pedagógicos y profesionales.

Para vislumbrar la efectividad de la implementación de políticas públicas, se requiere volver la mirada sobre la necesidad de repensar 
la importancia de volverse al maestro, como el protagonista en el proceso de reformas educativas en busca de una educación de calidad. De esta forma, es preciso que los países en sus propuestas educativas consideren e incorporen a los profesores y sus prácticas educativas logradas en el propio contexto escolar.

\section{Profesores y Reforma Educativa en Chile}

En el contexto de la Reforma Educacional que se ha llevado a cabo desde 1996, en Chile, se han implementado diferentes políticas orientadas al fortalecimiento de la profesión docente; entre otras acciones, se pueden mencionar programas de perfeccionamiento para dar a conocer a los docentes los elementos fundantes del nuevo currículo y las características de los distintos programas de estudio, pasantías al extranjero que han posibilitado a los profesores actualizarse en centros académicos de excelencia.

Al examinar la situación y participación de los profesores en Chile en los procesos de la Reforma Educacional antes mencionada, se puede señalar que, en un principio, en la reforma se distinguieron cuatro ámbitos de intervención, ellos eran:

I. Programas de mejoramiento e innovación,

2. Reforma curricular,

3. Desarrollo profesional de los docentes, y

4. Jornada escolar completa (Mineduc, 1998).

Lo anterior permitía, alentar expectativas respecto a participación y protagonismo de los profesores en dichos ámbitos, particularmente en los ámbitos segundo (Reforma curricular) y tercero (Desarrollo profesional de los docentes), cuando se señalaba:

Es una reforma que pretende afectar paulatina y en forma global todas las dimensiones del sistema: las formas de enseñar y aprender; los contenidos de la educación, la gestión de los servicios educativos, los insumos tanto de materiales educativos (bibliotecas, informática educativa) como de infraestructura 
escolar, el financiamiento del sector, así como el mejoramiento sostenido de las condiciones de trabajo de los docentes, principales artífices y protagonistas de la Reforma (Mineduc, I998: p. 24).

Si bien se reconocen los esfuerzos ministeriales por propiciar el fortalecimiento de la profesión docente, con la oferta de estrategias de formación continua distintas a las tradicionales como son los talleres comunales, las pasantías comunales, nacionales e internacionales, la constitución de la Red Maestro de Maestros, los docentes señalaban en una encuesta nacional (2003) que los aspectos que más han contribuido al fracaso de la reforma educativa es "la toma de decisiones técnicas inadecuadas a la realidad escolar" (22\%) y "bajo nivel de participación de profesores en la implementación” (I7\%).

... ambas opiniones dan cuenta de la falta de legitimidad de nuestro proceso de reforma, sin duda un aspecto a fortalecer si se espera generar el impulso necesario para un mayor número de escuelas efectivas (Raczynski y Muñoz, 2007).

Desde la percepción de los profesores, queda claro que se han sentido inmersos en una Reforma Educacional que no los consideró ni en su diseño ni en su implementación. Los logros que los profesores reconocen en la Reforma son para otros actores, como los niños y los jóvenes, pero hacia ellos distinguen más bien problemas, particularmente tienen una visión crítica hacia la Jornada Escolar Completa (JEC), porque ésta ha afectado su labor, aún cuando la valoran, señalan que las horas no se utilizan productivamente.

Otro aspecto de la Reforma Educacional que se destaca por parte de los profesores, es el de innovación tecnológica, aun cuando en profesores mayores la adaptación ha tenido algunas dificultades. En este contexto, a los profesores les preocupa la inequidad en la educación que se expresa en "la brecha digital", por lo que significa en tanto una "nueva" forma de desigualdad y segmentación social (Kuzmanich, 2004). Los profesores perciben que la Reforma educacional traería mayor segmentación y estratificación social, ya que 
no hay igualdad de oportunidades en el acceso ni en la permanencia, en instituciones que ofrezcan educación de calidad.

Por otra parte, el Ministerio de Educación considera que el cambio central es el de currículum, atendiendo a sus nuevos énfasis, puestos más en el desarrollo de capacidades y calidad de los aprendizajes. Los profesores respecto de este punto, perciben que una cosa es tener un nuevo currículum y otra cosa es implementarlo, una cosa es ampliar el tiempo escolar y otra cosa es usar ese tiempo útilmente... solo ha cambiado el currículum en los nombres, pero en el fondo es lo mismo (Kuzmanich, 2004).

Considerando el tema de la modernización, los docentes señalan que no solamente se debe modernizar la infraestructura, ya que eso mecánicamente no significa una modernización ni un mejoramiento en el sistema educacional, también deben considerarse en la Reforma aspectos sociales y culturales (Kuzmanich, 2004), o elementos subjetivos o simbólicos (Raczynski y Muñoz 2007).

Finalmente, los profesores comparten que el sistema educativo chileno requería una Reforma Educacional, las visiones críticas o discrepancias en algunos casos tienen que ver con el cómo ésta se ha gestado o implementado... como se grafica en el caso siguiente planteado por Kuzmanich:

El hacer tanto establecimiento para figurar en una cierta cantidad de tiempo, sólo para decir "en este período de tiempo se han construido tantos establecimientos educacionales por la Reforma Educacional"; creo que si se hubiese consultado desde las bases, tal vez no se hubiese politizado, porque yo hasta cierto punto veo la Reforma como una cosa política, pero la veo como una cuestión que es buena y la molestia que me causa es que a nosotros se nos impuso una reforma, pero de todas maneras la Reforma era necesaria independiente del gobernante, en eso no tengo ninguna duda; hubiese sido quien hubiese sido, nuestra educación necesitaba una reforma, necesitaba un cambio (Caso 8, mujer, 33 años de docencia en Kuzmanich, 2004: p. I08). 
De tal manera, que los profesores chilenos no es que se hayan opuesto a la Reforma educacional, sino que reconociendo que ella ha tenido logros, más bien, tienen una percepción crítica respecto a cómo se gestó e implementó, ya que se vivió como un proceso impuesto en el cual no se les consideró adecuadamente.

Considerando lo que ha sido la participación de los profesores en los procesos de Reforma educacional en Chile, encontramos un estudio (Raczynsky y Muñoz, 2007), en donde se señala, que teniendo como referente teórico las escuelas efectivas, que agregan valor al aprendizaje de sus estudiantes, se pueden visualizar las siguientes políticas educacionales que permitirían el mejoramiento de la educación en nuestro país:

I. Se debe pasar desde la macropolítica a la micropolítica desde la escuela, lo que significa que cualquier intervención o programa que se quiera implementar debe pensarse y realizarse desde la unidad educativa, ya que la Reforma ha privilegiado un diseño estandarizado y más bien rígido que no permite ni deja espacios a situaciones particulares ni participación de directivos y docentes.

2. Fortalecer el rol de los actores principales del cambio educativo: los profesores directivos y de aula. Centrar la atención en los profesores, se señala, significa ir tendiendo puentes entre los profesores y la reforma educativa, ya que el gremio docente no se ha sentido partícipe de la Reforma de los años '90, lo que ha desencadenado un involucramiento débil de los docentes en la misma.

3. Fortalecer el rol y las capacidades de los sostenedores. Uno de los principales temas a abordar en el sistema educativo chileno es el fortalecimiento de los soportes institucionales para posibilitar la efectividad escolar, lo cual resulta altamente relevante particularmente en el ámbito de sostenedores, en el mundo municipal como particular subvencionado.

4. Transitar desde un Ministerio de Educación ejecutor a uno que regula, evalúa y ofrece estrategias de apoyo diferenciadas. Se 
propone que para avanzar en la consecución de calidad y equidad en educación, el Ministerio de Educación debiera priorizar por evaluar el nivel de avance del sistema educativo (con foco en procesos de mejoramiento), regular y controlar el trabajo de los distintos actores (velando por la calidad de las prestaciones de los colegios particulares subvencionados, por ejemplo), y proveer apoyo técnico y programático en aquellos contextos donde no es posible crear capacidad local o donde las condiciones socioeconómicas de la población así lo exigen (Raczynski y Muñoz, 2007).

5. Fortalecer la responsabilización por resultados y rendición de cuentas en el sistema educativo. Para abordar lo anterior, se requiere mejorar los flujos de información y comunicación relevante hacia todos los agentes del sistema.

6. Favorecer una mayor equidad: subvención preferencial y escuelas socialmente diversas.

Se requiere en el sistema educativo chileno, crear escuelas socialmente más diversas y establecer una subvención diferenciada según la situación social del alumno.

\section{Reflexiones Finales}

Realizando una comparación con relación a la participación de los profesores en la Reformas Educativas de Brasil y Chile, se debe previamente observar qué caracterizó a ambos procesos.

En la Reforma Educativa brasileña se realizó una gran inversión pública, por ejemplo en formación y evaluación continua de profesores, se descentralizaron los aspectos administrativos-financieros, promoviéndose la autonomía escolar, pero por contraparte, colocando mayor regulación y control sobre los profesores. Otro aspecto que se puede señalar, es que se percibe mucha desinformación hacia los directores generando cierta impotencia por parte de ellos.

En la Reforma Educativa chilena, también al igual que en Brasil, se realizó una gran inversión pública, en formación inicial y continua de profesores, en infraestructura de colegios, en instalación de 
plataformas digitales, en una progresiva implementación de la Jornada Escolar Completa (JEC), entre otros. Se realizó un cambio de currículum en todo el sistema escolar. Quedan aún ciertos desafíos que resolver, al menos en nuevas legislaciones se empiezan a evidenciar, como son cambiar el foco de atención desde la macropolítica hacia la micropolítica educacional, el fortalecimiento del rol de los directivos docentes y profesores de aula, el fortalecimiento del rol de los sostenedores, favorecer una mayor equidad educacional, entre otros.

En síntesis, guardando las debidas diferencias entre ambas Reformas Educativas por las magnitudes de los recursos involucrados e impacto producido, en ellas se evidencia que los profesores no han sido debidamente considerados en las Reformas Educativas, de tal manera que se impone mejorar la calidad de los sistemas educativos de Brasil y Chile, en los cuales se han impulsado las reformas educativas, debiéndose considerar como condición de posibilidad y más bien incorporar protagónicamente a los profesores, reconociéndolos como actores centrales en el proceso de la reforma educativa.

Los profesores necesitan ser considerados, como sujetos centrales que tienen conocimientos, destrezas y experiencias que constituyen un saber pedagógico propio de los profesionales de la educación, que requiere ser considerado, valorado y puesto al servicio de la revitalización de la escuela pública de calidad. Así, ellos podrán comprometerse con el mejoramiento de la calidad de los aprendizajes de sus estudiantes, considerando las transformaciones del mundo y de la escuela, volviendo a crear categorías propias para elaborar un proyecto educativo de calidad, que rechace el dogmatismo de la retórica neoliberal, de modo de garantizar una escuela pública de calidad para todos, en búsqueda de una sociedad más justa y solidaria.

Finalmente, en Brasil y en Chile, considerando las diferencias -y las semejanzas- de los contextos políticos en que se han desarrollado sus transformaciones económicas, sociales y culturales, parece ser que el paradigma de las escuelas efectivas que agregan valor al aprendizaje, 
son un buen referente que permita fortalecer el rol de los profesores y los docentes directivos, en donde las escuelas puedan crecientemente ir tomando decisiones y eligiendo los apoyos más pertinentes a su realidad educativa, de tal manera que se transformen en escuelas efectivas a partir de docentes efectivos (Raczynski y Muñoz, 2007). En este sentido, es necesario seguir profundizando las políticas y acciones que fortalezcan el desarrollo profesional docente, ya que aún persisten ciertas insuficiencias tanto en la formación inicial como continua, de Profesores en servicio (Beca, 2006).

Sigamos entonces, invirtiendo en la búsqueda de la efectiva participación de los profesores en este contexto de cambio educativo, de forma que se conviertan en actores de las reformas educativas y no simples sujetos de políticas ajenas; para ello todas las iniciativas que promuevan el desarrollo profesional docente y el mejoramiento de los aprendizajes de los niños y niñas de ambos países son bienvenidas.

\section{Bibliografía}

Anderson, P. (1995). "Balanço do neoliberalismo". En Sader, E. \& Gentili, P. (orgs) Pós neoliberalismno. As políticas sociais e o estado democrático (pp.9-37). Rio de Janeiro, Paz e Terra.

Bianchetti, R. G. (1997). Modelo neoliberal e políticas educacionais. São Paulo: Cortez Editora.

Beca, C. E. et al., (2006). Docentes para el Nuevo siglo. Hacia una política de Desarrollo Profesional Docente. Serie Bicentenario, Ministerio de Educación.

Conferencia Episcopal de Chile. Orientaciones pastorales 2002-2005.

Esteve, J. M. (1995). “Mudanças sociais e função docente”. In Nóvoa, A (org.). Profissão professor (pp. 93-124). Porto Editora, Portugal.

Gentili, P. (1996). "Neoliberalismo e educação: manual do usuário”. In Silva, T. T. da \& GENTILI, P. (org.) Escola S. A. Brasília: CNTE.

Giroux, H. A. (1997). Os professores como intelectuais: rumo a uma pedagogia crítica da aprendizagem; (trad. Daniel Bueno). Porto Alegre: Artes Médicas.

Hargreaves, A. (2003). Profesorado, cultura y postmodernidad. (Cambian los tiempos, cambia el profesorado). Madrid, Morata.

Kuzmanich, A. (2004). Reforma Educacional Chilena: Percepción de Profesores de Colegios 
municipales de la Región Metropolitana. Tesis para optar al título de Sociología. Universidad de Chile.

Mainardes, J. A. (200I). "Organização da escolaridade em ciclos: Ainda um desafio para os sistemas de ensino”. En Franco, C. (org.). Avaliação, ciclos e promoçãa na educação. Porto Alegre: Artmed Editora.

Ministerio de Educación (1998). Reforma en Marcha: Buena Educación para Todos.

Ministerio de Educación (2004). Marco para la buena enseñanza.

Nunes, C. M. F. (2004). O saber da experiência de professores de séries iniciais: Condições de Produção e Formas de Manifestação. Rio de Janeiro: PUC-Rio. Departamento de Educação.

Oliveira, D. A. (2003). "As reformas educacionais e suas repercussões sobre o trabalho docente”. En Oliveira, D. A. (org). Reformas educacionais na A. L. e os trabalbadores docentes. Belo Horizonte: Autêntica.

Oliveira, D. A. (2002). Mudanças na organização e na gestão do trabalho na escola. En Oliveira, D.; Rosar, M. F. F. (org.). Política e Gestão da Educação. Belo Horizonte: Autêntica.

Oliveira, D. A. (2000). Educação Básica: gestão do trabalbo e da pobreza. Petrópolis/ RJ: Vozes.

Oliveira, D. A. (I999). “As reformas em curso nos sistemas públicos de educação básica: empregabilidade e eqüidade social”. En Oliveira, D. \& Duarte, M. Política e trabalbo na escola: administração dos sistemas públicos de educação básica. Belo Horizonte: Autentica.

Raczinski, D. y Muñoz, G. (2007). "Reforma educacional chilena: El Difícil equilibrio entre la macro y la micropolítica”. Cieplan, Serie Estudios Socio /Económicos No $3 \mathrm{I}$.

Silva, T. T. da (1994). "A "nova" direita e as transformações na pedagogia da política e na política da pedagógica”. En SILVA, T. T. da (org.), Neoliberalismo, qualidade total e educação. Petrópolis: Vozes.

Torres, R. M. (1996). "Melhorar a qualidade da educação básica? As estratégias do Banco Mundial”. En Tomassi, L. (org.) O banco mundial e as politicas educacionais. São Paulo, Cortez Editora.

Teixeira, L. H. G. (I999). "Políticas públicas de educação e mudança nas escolas. Um estudo da cultura escolar”. En Oliveira, D. \& Duarte, M. Política e trabalbo na escola: administração dos sistemas públicos de educação básica. Belo Horizonte: Autêntica. 